\title{
Investigation on Parameters of Atmospheric Pressure Plasma Jet by Electrical and Optical Methods
}

\author{
H. B. Baniya, R. P. Guragain, G. P. Panta, S. S. Safaai, \\ S. Dhungana, G. Qin and D. P. Subedi
}

Journal of Nepal Physical Society

Volume 6, Issue 2, December 2020

ISSN: 2392-473X (Print), 2738-9537 (Online)

\section{Editors:}

Dr. Binod Adhikari

Dr. Bhawani Joshi

Dr. Manoj Kumar Yadav

Dr. Krishna Rai

Dr. Rajendra Prasad Adhikari

Mr. Kiran Pudasainee

JNPS, 6 (2), 50-56 (2020)

DOI: http://doi.org/10.3126/jnphyssoc.v6i2.34857

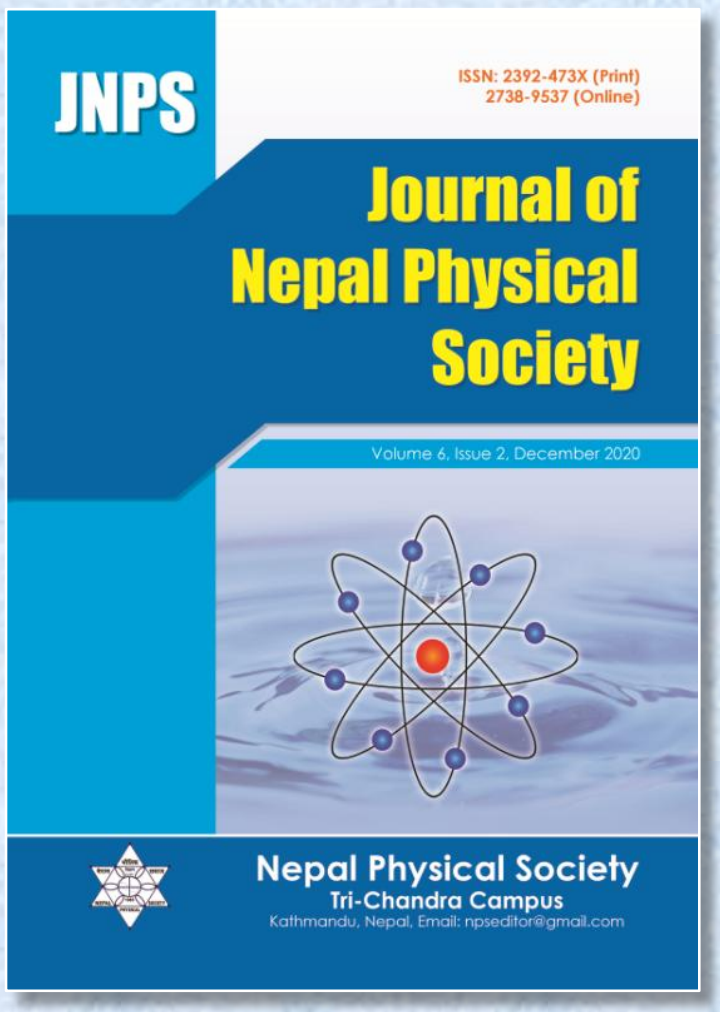

Published by:

Nepal Physical Society

P.O. Box: 2934

Tri-Chandra Campus

Kathmandu, Nepal

Email: npseditor@gmail.com 


\title{
Investigation on Parameters of Atmospheric Pressure Plasma Jet by Electrical and Optical Methods
}

\author{
H. B. Baniya ${ }^{1,2,}$, R. P. Guragain ${ }^{1}$, G. P. Panta ${ }^{1}$, S. S. Safaai ${ }^{3}$, S. Dhungana ${ }^{1}$, G. Qin $^{4}$ and D. P. Subedi $^{1}$ \\ ${ }^{1}$ Department of Physics, School of Science, Kathmandu University, Dhulikhel, Nepal. \\ ${ }^{2}$ Department of Physics, Tri-Chandra Multiple Campus, Tribhuvan University, Kathmandu, Nepal. \\ ${ }^{3}$ Department of Physics, Faculty of Science, University Technology Malaysia, Malaysia. \\ ${ }^{4}$ Harbin Institute of Technology, School of Science, Shenzhen, China. \\ *Corresponding Email: hombaniya@gmail.com, hom.baniya@trc.tu.edu.np
}

Received: 12 September, 2020; Revised: 15 November, 2020; Accepted: 28 December, 2020

\begin{abstract}
The atmospheric pressure plasma jet works under atmospheric pressure condition, has been developed for surface treatment and biomedical applications. The produced jet has been characterized by electrical and optical methods. To characterize cold atmospheric argon plasma discharge, its electron density, and electron energy (temperature) at various conditions have been estimated by using different techniques such as power balance, stark broadening, and intensity ratio methods respectively. Atmospheric pressure plasma jet (APPJ) has drawn much attention all over the world due to its applications in material processing, biomedical material processing, and thin film deposition. APPJ has been produced, using a high voltage and high frequency power supply $(0-20 \mathrm{kV})$ and an operating frequency of $20 \mathrm{kHz}$. Results showed that the electron density was of the order of $10^{14} \mathrm{~cm}^{-3}$ and $10^{16} \mathrm{~cm}^{-3}$ as determined by power balance, intensity ratio, and stark broadening methods respectively while electron temperature was estimated to be about $0.46 \mathrm{eV}$ and $0.53 \mathrm{eV}$ at $3 \mathrm{kV}$ and $4 \mathrm{kV}$ respectively by using intensity ratio method. The energy dissipation per cycle of the discharge was also estimated by using the Lissajous figure method. Our results confirmed that the parameter such as electron temperature and density depend on the applied voltage, gas flow rate, and electrode distance as well.
\end{abstract}

Keywords: Atmospheric pressure plasma jet, Electron temperature, Electron density, Energy dissipation, Electrical characterization, Optical characterization.

\section{INTRODUCTION}

The cold plasma discharge produced in atmospheric pressure is also stable even at low frequency as compared to RF sources which is much more expensive.

The most of the previous works have used RF power supply which is more expensive compared to the power supply used in the present study. The plasma jet is designed with locally available materials and can be made treatment continuous for a long time. We have successfully developed a plasma device that is able to generate nonequilibrium APPJ of low temperatures. The costeffective system of generating argon plasma jet at atmospheric pressure having different applications in materials processing and biomedical research has been developed $[1,2]$. Plasma is an ionized gas of charged and neutral particles which shows collective behavior. It is an electrically conductive gas containing charged particles. The atoms of process gas are excited to higher energy states, some of their electrons get ionized, producing plasma containing electrically charged particles, ions, and electrons. Langmuir proposed that the electrons, ions, and neutrals in an ionized gas could similarly be considered as corpuscular material entrained in some kind of fluid medium and called this fluid medium plasma [2]. However, it turned out that unlike blood where there really is a fluid medium carrying the corpuscular material, there actually is no fluid medium entering the electrons, ions, and neutrals in an ionized gas. 
Generally, plasma is the fourth state of matter after solid, liquid, and gas. The dynamic nature of the plume is investigated by using an intensified camera [3, 4]. To determine plasma parameters, atmospheric pressure cold argon plasma jet has been characterized electrically and optically with respect to applied voltage and frequency to understand the dynamic behavior of discharge. The plasma jet is generated with a capacitive coupled dielectric barrier discharge and a working gas of argon flowing out into the environmental air [5, 6]. The produced argon plasma jet is harmless for humans because of its low voltage [7]. In order to characterize the argon plasma jet, its electron temperature and its composition have been determined by means of optical emissions spectroscopy [7, 8]. Therefore, the plasma characterization techniques are important to determine the plasma conditions [9]. In the present experimental setup, three glass tubes are coupled using a nylon coupler which facilitates introducing an additional inlet for gases in the discharge regime without any detriment to the jet formation mechanism [10-12].

\section{MATERIALS AND METHODS}

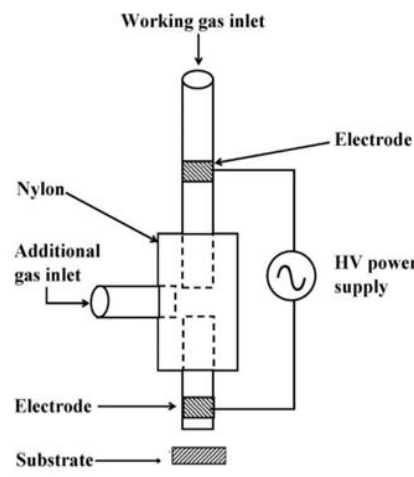

(A)

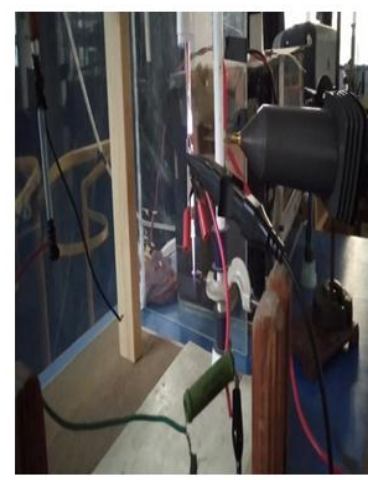

(B)
Fig. 1: (A)The experimental setup and (B) Image of the discharge along with voltage and current probes[16]

The experimental set up to produce plasma jet is shown in figure 1(A). The experimental set up consists of capacitive coupled electrode system made of copper foil of $3 \mathrm{~mm}$ diameter wrapped around two ordinary glass tube of outer diameter 50 $\mathrm{mm}$ and inner diameter $40 \mathrm{~mm}$. The copper electrode is connected to high voltage power supply and the glass tube is coupled with nylon coupler of length $5 \mathrm{~cm}$. The distance between two electrodes is fixed at $50 \mathrm{~mm}$ and the distance between the tip of the nozzle and lower electrode is $30 \mathrm{~mm}$. The system consists of one additional inlet with a third tube connected with nylon coupler horizontally in the middle as shown in figure 1 (A). The third inlet can be used to introduce additional gas into the system. During the experiment, argon was used as a main working gas in the experiment.

The flow rate for argon gas is 1-2 ltr/min and the voltage and the frequency were maintained at 3-4 $\mathrm{kV}$ and $20 \mathrm{kHz}$ respectively. The figure 1(B) shows the image of plasma jet along with current and voltage probes for electrical characterization. Figure1(B) shows the electrical characterization of the discharge which was done by measuring the current and voltage wave-forms, were analyzed in TEKTRONIX TDS2002 oscilloscope by using current probe and voltage probe (PINTEX HVP$28 \mathrm{HF}$ ) respectively. The attenuation ratio of the voltage probe is 1000:1. Similarly, Optical characterization of the discharge were done by using Line intensity ratio and Stark broadening methods with the help of optical emission spectrometer (USB 2000+, Ocean Optics). Materials used for the electrical and optical characterization of atmospheric pressure plasma jet are shown in figure 2 .

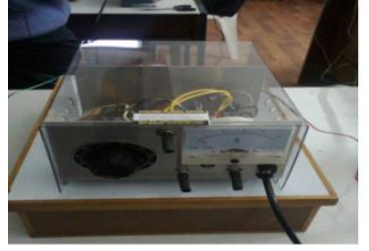

HV POWER SUPPLY

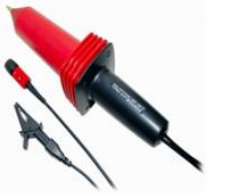

PINTEX HVP

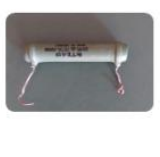

RESISTOR

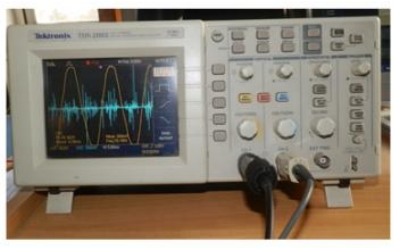

TEKTRONIX TDS 2002

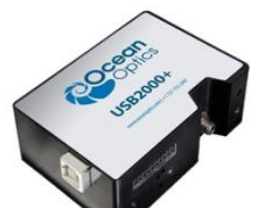

OPTICAL EMISSION SPECTROMETER
Fig. 2: Materials used during the experiment for electrical and optical characterization of APPJ

\section{RESULTS AND DISCUSSION}

Temperature determination of atmospheric pressure plasma jet (APPJ)

Figure 3 shows the temperature of plasma jet with time at discharge voltage $4 \mathrm{kV}$. It was linear up to 60 seconds then after almost remains constant from 90-180 seconds as shown in figure 3. Atmospheric pressure plasmas have pressure approximately matches with surrounding atmosphere. This plasma is also called normal plasma. The plasma jet was 


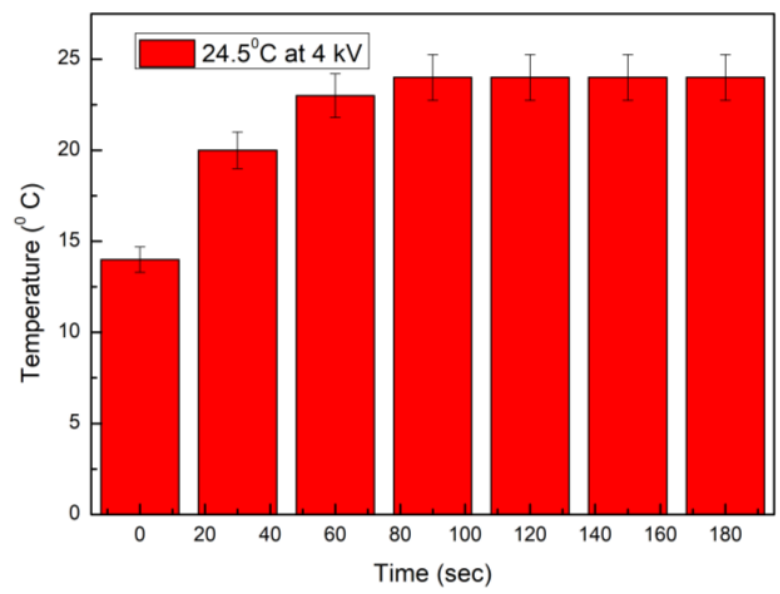

Fig. 3: Temperature measurement of APPJ at $4 \mathrm{kV}$ and an operating frequency of $20 \mathrm{kHz}$

designed with locally available materials to make treatment continuous and cost effective. Temperature of plasma jet was directly measured as working condition using thermometer is about $24.5^{0}$ $\mathrm{C}$ at working voltage $4 \mathrm{kV}$ and an operating frequency of $20 \mathrm{kHz}$. So that the produced plasma discharge sustained at low temperature is called cold atmospheric pressure plasma jet and widely used in biomedical applications [1].

\section{Electrical characterization of APPJ}

The total power absorbed by a capacitive coupled atmospheric pressure plasma jet can be written as $P_{a v}=2 A n_{e} v_{b} E_{\text {lost }}$ where, $2 A n_{e} v_{b} E_{\text {lost }}$ represents the total power of the discharge over the area $2 A$ of the two electrodes, $\mathrm{n}_{\mathrm{e}}$ being the electron density, $\mathrm{E}_{\text {lost }}$ being the energy lost per cycle, and $v_{b}$ being the Bohm velocity. The electron density is estimated using the power balance method expressed in the equation (1).

$$
n_{e}=\frac{P_{a v}}{2 A v_{b} E_{l o s t}}
$$

This equation can be used to determine the electron density in the glow mode of the discharge $[13,14]$.

Figure 4 (A) shows the discharge voltage with respect to time and 4(B) shows the discharge current with respect to time of APPJ generated in argon with electrode gap of $50 \mathrm{~mm}$, applied voltage $4 \mathrm{kV}$ and an operating frequency of $20 \mathrm{kHz}$ at atmospheric pressure condition.

Using the values of applied voltage and average discharge current, the electron density is determined using the equation (1). Using the value of Bohm velocity $v_{b}=2 \times 10^{3} \mathrm{~m} / \mathrm{s}$ [14], energy lost $80 \times 10^{-19} \mathrm{~J}$, electrodes area $4.096 \times 10^{-5} \mathrm{~m}^{2}$, applied voltage $4 \mathrm{kV}$ and discharge current about $20 \mathrm{~mA}$, the electron density $\left(\mathrm{n}_{\mathrm{e}}\right)$ was found to be $3.5 \times 10_{10}^{14} \mathrm{~cm}^{-3}$. The power $(\mathrm{P}=\mathrm{VI})$ consumed per cycle was about 80 watt [14-16].

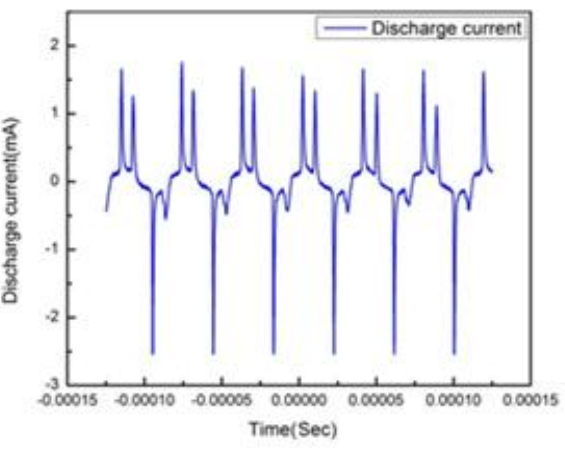

(B)

(A)

Fig. 4: (A) Voltage and (B) current waveforms as the function of treatment time at an applied voltage $4 \mathrm{kV}$ and an operating frequency of $20 \mathrm{kHz}$ (flow rate of $\operatorname{argon}(\mathrm{Q})=2 \mathrm{Itr} / \mathrm{min}$ )

\section{Determination of energy dissipation during each cycle of discharge}

Electrical characterization of atmospheric pressure plasma jet is carried out by connecting a voltage probe across the electrodes and current probe is connected along with suitable resistor of resistance $10 \mathrm{k} \Omega$. Energy dissipated is one of the parameter of produced atmospheric argon plasma jet. The energy produced depends on applied voltage, area 
of the electrodes and volume of gas flow rate and determined energy using lissajous figure as shown in figure 5 . The area covered by lissajous figure

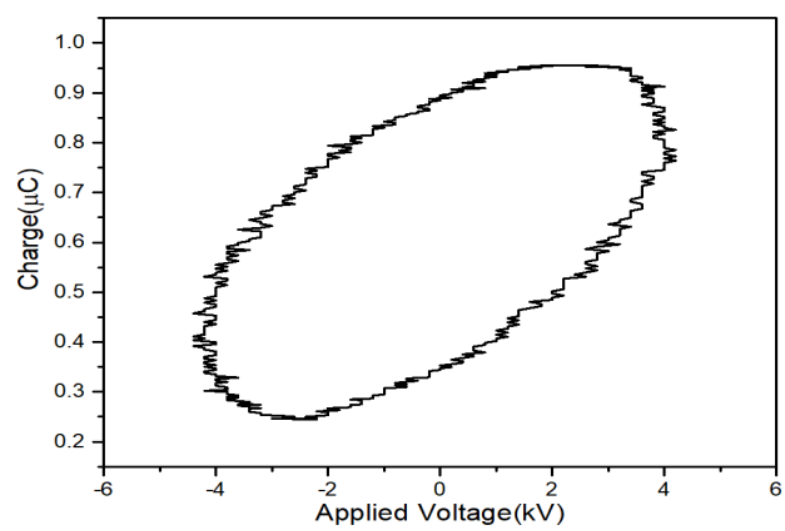

Fig. 5: Lissajous figure: plot of graph between charge and voltage $(4 \mathrm{kV})$

gives the total energy of the discharge in each cycle[13, 15]. Figure 5 shows the Lissajous figure plotted between the data of charge and applied voltage used to determine the total energy of cold plasma discharge in each cycle. Total area enclosed by lissajous figure is estimated about $3.6 \times 10^{-4} \mathrm{~m}^{2}$. We have, total energy of the discharge per cycle is equal to the total area enclosed by lissajous figure. Hence, the total energy dissipated during each cycle of discharge is $3.6 \times 10^{-4} \mathrm{~J}$.

\section{Optical characterization of APPJ}

The optical emission spectra of APPJ in the range of $200-1100 \mathrm{~nm}$ is shown in figures 6 and 7 . The resolution $0.1 \mathrm{~nm}$ of optical emission spectrometer (USB 2000+ from Ocean Optics) has been used to characterize plasma species present in the discharge. The most intensive $\mathrm{OH}$ line found at 309 $\mathrm{nm}$ and $282.6 \mathrm{~nm}$ shows line of least intensity indicating emission of UV radiation. The prominent argon lines are found in the wavelength range from $696 \mathrm{~nm}$ to $850 \mathrm{~nm}$ which indicates the different transition of argon lines. The peaks in the region $330 \mathrm{~nm}$ to $350 \mathrm{~nm}$ shows the transition of nitrogen.

The optical characterization of the discharge was carried out by using the line intensity ratio method. In this method four suitable lines (two for Ar I and two for Ar II) were chosen from spectral lines obtain from atmospheric pressure glow discharge(APGD). The working formula used to calculate the electron temperature is as follows $[17,18]$ :

$$
\frac{R_{1}}{R_{2}}=\frac{I_{1} / I_{2}}{I_{3} / I_{4}}=\left(\frac{A_{p q}}{A_{r s}}\right)\left(\frac{g_{p}}{g_{r}}\right)\left(\frac{\lambda_{r s}}{\lambda_{p q}}\right)\left(\frac{A_{u v}}{A_{x y}}\right)\left(\frac{g_{u}}{g_{x}}\right)\left(\frac{\lambda_{x y}}{\lambda_{u v}}\right) \exp \left[-\frac{E_{p}-E_{r}-E_{x}+E_{v}}{K_{B} T_{e}}\right]
$$

Here, in equation (2), $R$ is the ratio of the intensity of two lines, $\boldsymbol{I}$ is the intensity of the spectral line, $A_{j i}$ is the transition probability of the transition $i \rightarrow j, g_{i}$ is the statistical weight of the upper level, $\lambda$ is the wavelength of the line radiation, $E_{i}$ is the energy of the upper level, $K_{B}$

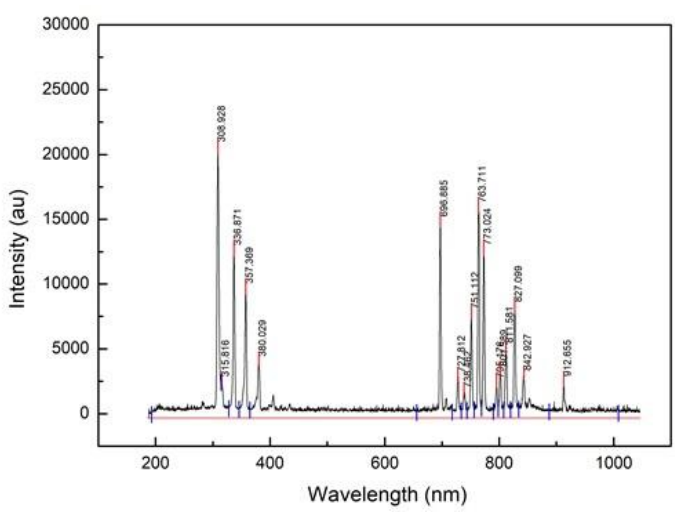

Fig. 6: Spectra of the discharge at frequency $20 \mathrm{kHz}$ and is Boltzmann constant and $T_{e}$ is the electron temperature. The values of $\lambda$ and $I$ are obtained from the observation, and the values of $A_{j i}, g_{i}$ and $E_{i}$ are obtained from the National Institute of Standards and Technology (NIST) Atomic Spectra Database [19].

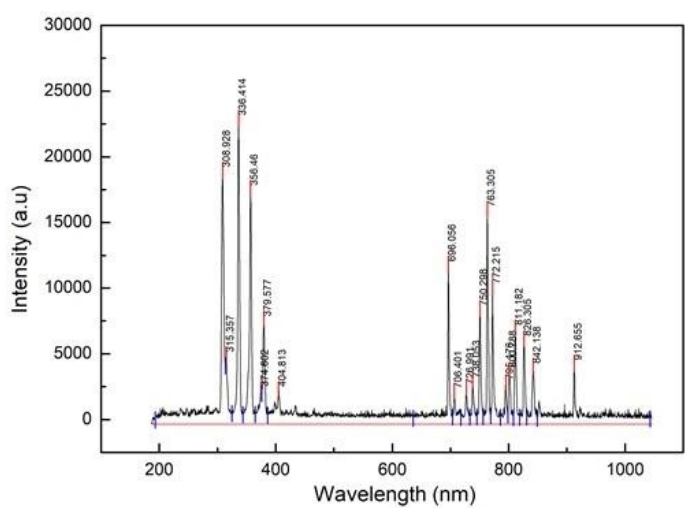

Fig. 7: Spectra of the discharge at frequency $20 \mathrm{kHz}$ and 
Investigation on parameters of atmospheric pressure plasma jet by electrical and optical methods

an applied voltage of $3 \mathrm{kV}$ in argon environmment( flow rate $=2 \operatorname{ltr} / \mathrm{min})$ an applied voltage of $4 \mathrm{kV}$ in argon environmment (flow rate $=2 \mathrm{Itr} / \mathrm{min})$

Table 1: Intensity and wavelength of Ar I and Ar II lines at $3 \mathrm{kV}$ and $4 \mathrm{kV}$

\begin{tabular}{|l|l|l|l|l|l|l|}
\hline Lines & \multicolumn{1}{|c|}{$\begin{array}{c}\text { Wavelength } \\
(\mathbf{n m})\end{array}$} & $\begin{array}{c}\text { Transition } \\
\text { probability } \\
\left(\mathbf{S}^{-1}\right)\end{array}$ & $\begin{array}{c}\text { Statistical } \\
\text { weight }\end{array}$ & Energy (eV) & $\begin{array}{c}\text { Intensity(au) } \\
\text { at } \mathbf{3 ~ k V}\end{array}$ & $\begin{array}{c}\text { Intensity(au) } \\
\text { at } \mathbf{4 ~ k V}\end{array}$ \\
\hline Ar I & 696.54 & $A_{p q}=6.39 \times 10^{6}$ & $g_{p}=5$ & $E_{p}=11.564$ & $\mathrm{I}_{1}=14103.43$ & $\mathrm{I}_{1}=16778$ \\
\hline $\operatorname{Ar} \mathrm{I}$ & 749.87 & $A_{r s}=4.02 \times 10^{7}$ & $g_{r}=3$ & $E_{r}=11.636$ & $\mathrm{I}_{2}=8317.32$ & $\mathrm{I}_{2}=8954.44$ \\
\hline Ar II & 314.82 & $A_{x y}=5.20 \times 10^{7}$ & $g_{x}=6$ & $E_{x}=19.249$ & $\mathrm{I}_{3}=5720.66$ & $\mathrm{I}_{3}=6211.09$ \\
\hline Ar II & 379.81 & $A_{u v}=1.05 \times 10^{6}$ & $g_{u}=8$ & $E_{u}=16.797$ & $\mathrm{I}_{4}=8227.50$ & $\mathrm{I}_{4}=8201.75$ \\
\hline
\end{tabular}

Table 1 shows the particular wavelengths and their corresponding values of different parameters. On substituting these values obtained from the National Institute of Standards and Technology (NIST) Atomic Spectra Database [19] in equation (2), the ratio of the intensities is expressed as in equation(3).

$$
\frac{R_{1}}{R_{2}}=1.14 \times 10^{-2} \exp \left[\frac{2.524}{k T_{e}}\right]
$$

At different values of electron temperature, the values of ratio of intensities of the corresponding wavelength were determined. When graph plotted between the data of intensities ratio and corresponding electron temperature, the nature is as shown in figure 8 .

The ratio of intensity of these four particular wavelengths $\left(\frac{I 1 / I 2}{I 3 / I 4}\right)$ was 2.4 at $3 \mathrm{kV}$ and 1.17 at 4

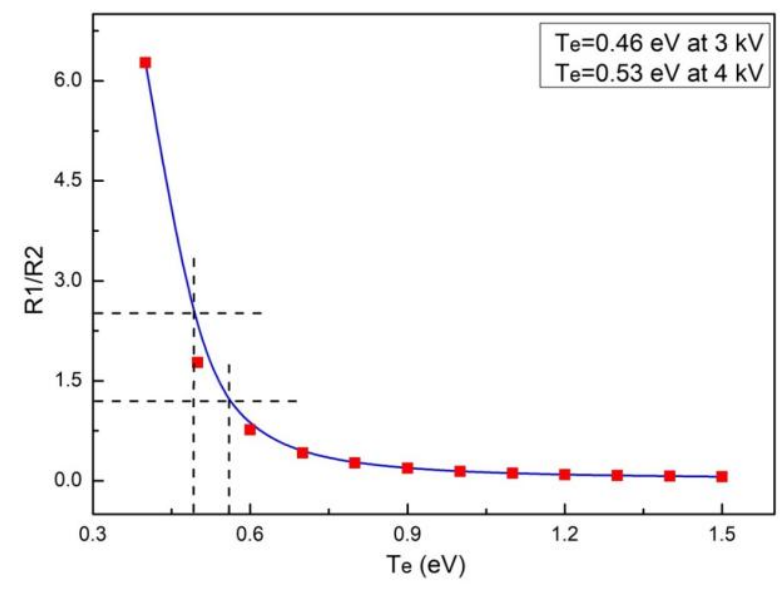

Fig. 8: Graph of intensities ratio of corresponding wavelength as the function of electron temperature

$\mathrm{kV}$. Hence, from the graph, the electron temperature $\left(\mathrm{T}_{\mathrm{e}}\right)$ was found to be $0.46 \mathrm{eV}$ and 0.53 $\mathrm{eV}$ at $3 \mathrm{kV}$ and $4 \mathrm{kV}$ respectively.

Similarly, from both the cases, the electron density were calculated by using equation (4).

$$
n_{e}=2\left(\frac{I_{1}}{I_{2}}\right)\left(\frac{\lambda_{1}}{\lambda_{2}}\right)\left(\frac{A_{2}}{A_{1}}\right)\left(\frac{g_{2}}{g_{1}}\right)\left(\frac{2 \pi m_{e} k T_{e}}{h^{2}}\right)^{3 / 2} \exp \left(\frac{-E_{1}-E_{2}+E_{i}}{k T_{e}}\right)
$$

Substituting these parameters obtained from the National Institute of Standards and Technology (NIST) Atomic Spectra Database [19] in equation (4), the electron density was found to be $1.82 \times$ $10^{16} \mathrm{~cm}^{-3}$ and $2.11 \times 10^{16} \mathrm{~cm}^{-3}$ at $3 \mathrm{kV}$ and $4 \mathrm{kV}$ respectively.

\section{Stark Broadening Method}

The emission spectra of the discharge were further used to measure electron density $\left(\mathrm{n}_{\mathrm{e}}\right)$ using Stark broadening method. In this method the argon line of $696.54 \mathrm{~nm}$ was chosen. By finding the value of $\Delta \lambda_{\text {stark }}$ using FWHM and substituting in equation (5) 
we obtained values of $n_{e}$ for different plasma conditions. The dependence of $\mathrm{n}_{\mathrm{e}}$ on different plasma conditions was determined as fellows [16, 20, 21]. The electron density is calculated by full width half maximum (FWHM) according to the relation represented by equation (5).

$$
\Delta \lambda_{\text {Stark }}=2 \times 10^{-11} n_{e}^{2 / 3}
$$

\section{Effect of applied Voltage}

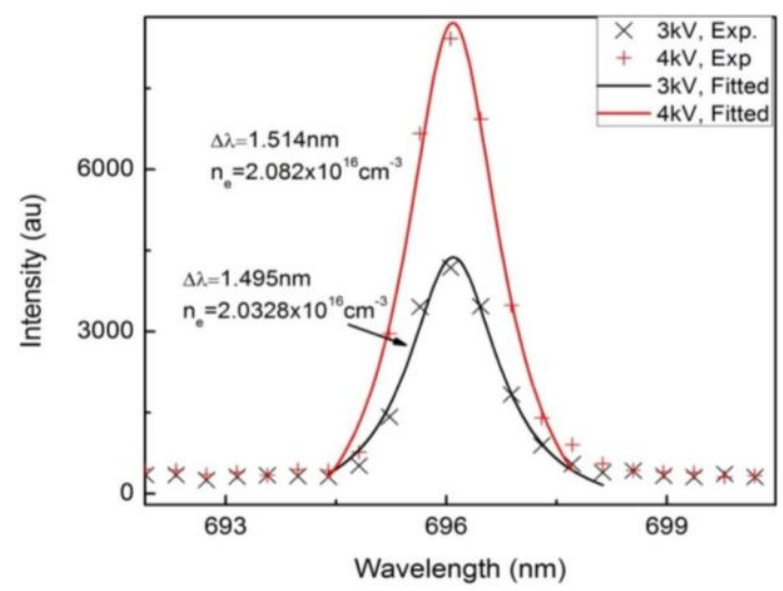

Fig. 9: Comparison of electron density of plasma jet at working voltage $3 \mathrm{kV}$ and $4 \mathrm{kV}$ (argon flow rate $2 \mathrm{ltr} / \mathrm{min}$ and an operating frequency of $20 \mathrm{kHz}$ ).

Figure 9 shows the stark broadening of the line at $696.54 \mathrm{~nm}$ gives a reasonable approximation of the electron density of the discharge. Lorentzian fit of the data for the line is shown in figure 9. Spectral line analysis is a good method for determining electron density. The electron density is related to FWHM according to the relation represented by equation (3). The value of $\Delta \lambda_{\text {stark }}$ is $1.49 \mathrm{~nm}$ and the corresponding electron density is $2.032 \times 10^{16} \mathrm{~cm}^{-3}$ when the applied voltage was $3 \mathrm{kV}$. Similarly, $\Delta \lambda_{\text {stark }}$ is $1.51 \mathrm{~nm}$ and the corresponding electron density is $2.08 \times 10^{16} \mathrm{~cm}^{-3}$ when the applied voltage was $4 \mathrm{kV}$. It is seen from the result that the electron density is proportional to the applied voltage.

\section{Effect of gas flow rate}

Figure 10 shows the stark broadening of the line at $696 \mathrm{~nm}$ gives a reasonable approximation of the electron density of the discharge. Lorenzian fit of the data for the line is shown in figure 10. Spectral line analysis is a good method for determining electron density. The electron density is related to FWHM according to the relation represented by equation (3). The value of $\Delta \lambda_{\text {stark }}$ is $1.448 \mathrm{~nm}$ and the corresponding electron density is $1.948 \times 10^{16}$ $\mathrm{cm}^{-3}$ when the argon gas flow rate was $1 \mathrm{ltr} / \mathrm{min}$. Similarly, $\Delta \lambda_{\text {stark }}$ is $1.508 \mathrm{~nm}$ and the corresponding electron density is $2.07 \times 10^{16} \mathrm{~cm}^{-3}$ when the gas flow rate was $2 \mathrm{ltr} / \mathrm{min}$. It is seen from the result that the electron density is proportional to the flow rate of gas $[20,21]$.

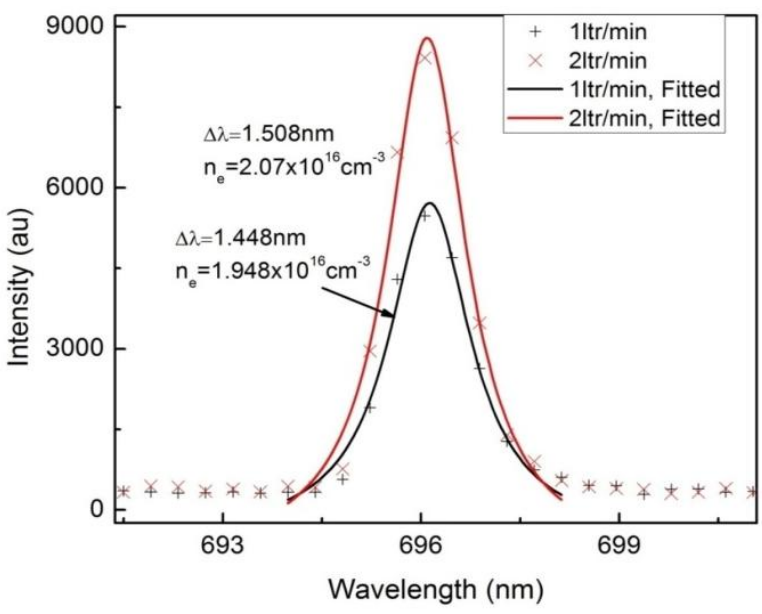

Fig. 10: Comparison of electron density of plasma jet of process gas (argon flow) $1 \mathrm{ltr} / \mathrm{min}$ and $2 \mathrm{ltr} / \mathrm{min}$ (at $4 \mathrm{kV}$ and an operating frequency of $20 \mathrm{kHz}$ )

\section{CONCLUSION}

The cost-effective system of generating plasma jet at atmospheric pressure with potential applications in material processing has been developed. The temperature of plasma jet was to be measured about $24.5^{0} \mathrm{C}$ at $4 \mathrm{kV}$ so that the discharge is termed as cold plasma. Atmospheric pressure argon plasma jet has been produced and estimated plasma parameters by optical and electrical methods. Electron density $\left(\mathrm{n}_{\mathrm{e}}\right)$ and electron temperature $\left(\mathrm{T}_{\mathrm{e}}\right)$ were found to be of the order of $10^{14} \mathrm{~cm}^{-3}$ and $10^{16}$ $\mathrm{cm}^{-3}[16]$ using power balance, intensity ratio and stark broadening methods and $0.46 \mathrm{eV}$ and $0.53 \mathrm{eV}$ [10] at applied voltage $3 \mathrm{kV}$ and $4 \mathrm{kV}$ respectively using intensity ratio method. Energy dissipated each cycle of discharge was found to be $3.6 \times 10^{-4} \mathrm{~J}$, using the Lissajous figures method. It was found that electron temperature increases when applied voltage increased. It has been seen from the results that electron density was proportional to applied voltage and gas flow rate as well.

\section{ACKNOWLEDGEMENT}

This research was partially supported by Nepal Academy of Science and Technology (NAST), 
Nepal, providing $\mathrm{PhD}$ Fellowship through Grant No: 11/073/074.The authors would like to acknowledge Department of Physics, School of Science, Kathmandu University, Department of Physics, Tri-Chandra Multiple Campus (T.U.) and Institute of Science and Technology (IOST), Tribhuvan University, Nepal for their invaluable help and support.

\section{REFERENCES}

[1] Baniya, H. B.; Guragain, R. P.; Baniya, B. and Subedi, D.P. Experimental study of cold atmospheric pressure plasma jet and its application in the surface modification of polypropylene. Reviews of Adhesion and Adhesives, 8(2): S1-S14 (2020.

[2] Chen, F. F.; Evans, J. D.; and Zawalski, W. Electric Probes, In Plasma Diagnostic Techniques. R. H. Huddle stone, and S. L. Leonard (Eds.), Chapter 4 (113-200). New York, USA: Academic Press (1965).

[3] Park, H. S.; Kim, S. J.; Joh, H. M.; Chung, T. H.; Bae, S. H.; and Leem, S. H. Optical and electrical characterization of an atmospheric pressure micro plasma jet with a capillary electrode, Physics of Plasmas, 17: 033502 (2010).

[4] Baniya, H. B.; Shrestha, S. and Subedi, D. P. Effect of floating electrode on the length of the capacitively coupled atmospheric pressure plasma jet. International Journal of Engineering Research-Online; a Peer Reviewed International Journal, 4(1): 163-167(2016).

[5] Marinov, D. and Braithwaite, N. St J. Power coupling and electrical characterization of a radio-frequency micro atmospheric pressure plasma jet. Plasma Sources Science and Technology, 23 (6): 062005 (2014).

[6] Shrestha, A. K.; Shrestha, R.; Baniya, H. B.; Tyata, R. B.; Subedi, D. P. and Wong, C. S. Influence of discharge voltage and pressure on the plasma parameters in a low pressure DC glow discharge, International Journal of Recent Research and Review, 7 (2): (2014).

[7] Fester, S.; Mohr, C. andViöl, W. Investigations of atmospheric pressure plasma jet by optical emission spectroscopy, Surface and Coatings Technology, 4 (200): 827-830 (2005), https://doi. org/10.1016/j.surfcoat.2005.02.217.

[8] Pavia, D. L.; Lampman, G. M. and Kriz, G. S. Introduction to Spectroscopy, Sanat Printers, 3rd edition (2001); https: //trove.nla.gov.au/work/ 8887496.

[9] Konuma, M. Film deposition by plasma techniques, Springer Science and Business Media, 10: 14-18 (2012).
[10] Baniya, H. B.; Shrestha, R.; Guragain, R. P.; Kshetri, M. B.; Pandey, B. P. and Subedi, D. P. Generation and characterization of an atmospheric pressure plasma jet (APPJ) and its application in the surface modification of polyethylene terephthalate. International Journal of Polymer Science, 2020: 1-7 (2020).

[11] Das, S.; Das, D. P.; Sarangi, C. K.; Bhoi, B.; Mishra, B. K.; and Ghosh, J. Optical emission spectroscopy study of $\mathrm{Ar}-\mathrm{H}_{2}$ plasma at atmospheric pressure. IEEE Transactions on Plasma Science, 46(8): 2909-2915 (2018).

[12] Baniya, H. B.; Guragain, R. P.; Baniya, B.; Qin, G. and Subedi, D. P. Improvement of hydrophilicity of polyamide using atmospheric pressure plasma jet. J. Physical Sciences, 17: 133-138 (2020).

[13] Trusov, K. K. Dynamics of multichannel and quasi-homogeneous sliding discharge formation in rare gases. J. Phy. D: Appl. Phy. 40: 786-794 (2007).

[14] Wong, C. S. and Mongkolnavin, R. Plasma diagnostics techniques, In: Elements of Plasma Technology. Springer Briefs in Applied Sciences and Technology, Springer (2016).

[15] Balcon, N.; Aanesl, A. and Boswell, R. Pulsed RF discharges, glow and filamentary mode at atmospheric pressure in argon. Plasma Sources Science and Technology, 16 (2): 217 (2007).

[16] Baniya, H. B.; Guragain, R. P.; Baniya, B., and Subedi, D. P. Cold atmospheric pressure plasma jet for the improvement of wettability of polypropylene. International Journal of Polymer Science, 2020: 1-9 (2020).

[17] Falahat, A.; Ganjovi, A.; Taraz, M.; Ravari, M. N. R. and Shahedi, A. Optical characteristics of a $\mathrm{RF}$ DBD plasma jet in various $\mathrm{Ar} / \mathrm{O}_{2}$ mixtures. Pramana - J. Physics, 90: 27 (2018).

[18] Sarani, A.; Nikiforov, A. and Leys, C. Atmospheric pressure plasma jet in $\mathrm{Ar}$ and $\mathrm{Ar} / \mathrm{H}_{2} \mathrm{O}$ mixtures: optical emission spectroscopy and temperature measurements. Physics of Plasmas, 17: 063504 (2010).

[19] Kramida, A.; Ralchenko, Y.; Reader, J. and NIST ASD Team. NIST Atomic Spectra Database, Online: https://physics.nist.gov/asd [2019, March 14], 2018.

[20] Baniya, H. B. Experimental study of atmospheric pressure plasma jet with floating electrode and its application for polymer surface modification. M. Phil. Thesis, Kathmandu University, Nepal, 2016.

[21] Dong, L.; Ran, J. and Mao, Z. Direct measurement of electron density in microdischarge at atmospheric pressure by stark broadening. Applied Physics Letters, 86: 161501 (2005). 